\title{
A Two-Fingered Robot Gripper with Large Object Reorientation Range
}

\author{
Walter G. Bircher, Student Member, IEEE, Aaron M. Dollar, Senior Member, IEEE, \\ and Nicolas Rojas, Member IEEE
}

\begin{abstract}
It is very challenging for a robotic gripper to achieve large reorientations with grasped objects without accidental object ejection. This paper presents a simple gripper that can repeatedly achieve large reorientations over $\pi / 2 \mathrm{rad}$ through the kinematics of the hand-object system alone, without the use of high fidelity contact sensors, complex control of active finger surfaces, or highly actuated fingers. This gripper is the result of two kinematic parameter search optimizations connected in cascade. Besides the large range of reorientation attained, the obtained gripper also corresponds to a novel topology since ternary joints in the palm are presented. The inhand planar reorientation capabilities of the proposed gripper are experimentally tested with success.
\end{abstract}

\section{INTRODUCTION}

One of the most challenging problems in robotic manipulation is reorienting an object while maintaining a stable grasp [1]. For fully actuated robotic graspers, reorienting an object might require precisely planned regrasp with some fingers, while others maintain hold of the object. This type of motion is not possible without carefully balancing forces on an object, and without some knowledge of the object's shape [2]. Furthermore, force balance is highly dependent on precise contact locations, requiring high-fidelity contact sensors - an open research problem in itself [3]. Active surfaces, such as finger mounted turntables or conveyor belts, are a useful approach to reorientation, but the motion of opposing surfaces must still be choreographed to avoid excessive object translation from rolling and sliding (e.g., [4]). Thus, in order to create reorientation that is repeatable, openloop, and does not depend on careful force balance or active surface coordination, we turn to manipulation behavior achieved through the kinematics of the hand itself.

The 'grasp-reposition-reorient gripper,' known as the GR2 gripper, is a two-finger grasper whose hand-object system generates a reconfigurable Innocenti linkage, which connects two four bar mechanisms through a central pivoting link, coupling their motion together [5]. The GR2 is worthy of study because of its ability to perform in-hand manipulation without significant additional control complexity. Additionally, the GR2 gripper has a unique central pivot that can either be locked in place or supported by elastic bands to produce different reorientation behaviors.

Many researchers have leveraged unique mechanisms to enhance in-hand manipulation capabilities of robotic grippers. Bicchi et al. constructed a two finger gripper with opposing

This work was supported in part by the National Science Foundation grants IIS-1317976 and IIS-0952856.

W. G. Bircher and A. M. Dollar are with the Department of Mechanical Engineering and Materials Science, Yale University, New Haven, CT, USA. (e-mail: \{walter.bircher; aaron.dollar\}@yale.edu)

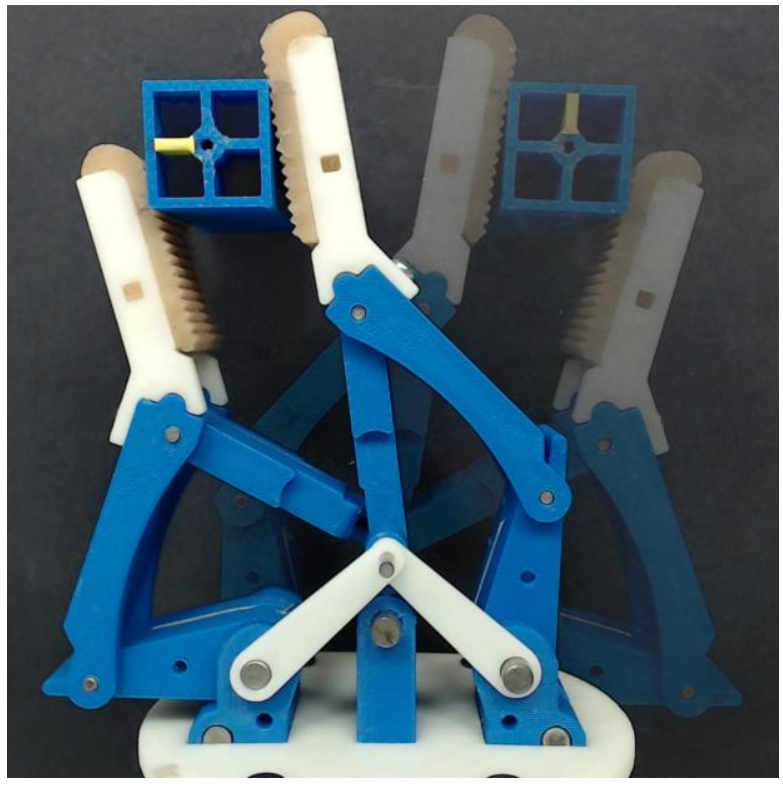

Figure 1. The coalesced GR2 gripper. This simple two-finger robot gripper with a ternary joint in the palm is able to reorient objects in excess of $\pi / 2 \mathrm{rad}$ while maintaining a stable grasp, without using highfidelity contact sensors, active/sliding finger surfaces, or a priori workspace exploration. Each finger is independently actuated.

turn-tables, that perform in-hand manipulation purely through rolling [6]. Tincani et al. developed a gripper with conveyorbelt surfaces on each finger that is also able to manipulate an object through rolling [7]. Odhner et al. used an underactuated grasper to robustly and repeatedly perform grasp primitives, such as pinch grasps and power grasps, as well as in-hand manipulation tasks such as grasp transitions [8]. A four finger underactuated hand was used by $\mathrm{Ma}$ et al. to perform fingergaiting, and even continuous in-hand twisting [9]. However, these hands are all limited in the amount of reorientation they can achieve without regrasp or changing contact conditions. Conversely, the GR2 specifically leverages underactuation to increase the range of reorientation it can apply to a grasped object [5].

There is a substantial volume of literature related to kinematic optimization of mechanisms, a large portion of which consists of grasper optimization. Ciocarlie et al. optimize an underactuated gripper to achieve enveloping grasps for a large range of objects, using random search and gradient descent techniques [10]. Rodríguez, Carbone, and

N. Rojas is with the Dyson School of Design Engineering, Imperial College London, London SW7 1NA, UK. (e-mail: n.rojas@imperial.ac.uk). 
Ceccarelli synthesize the driving mechanism of a $1-\mathrm{DoF}$ anthropomorphic finger, by minimizing the difference between a cubic interpolation of data obtained from measuring human finger motion, and a function approximating the motion of the grasper finger linkage [11].

This paper is organized as follows: Section II explains the formulation of the linkage used to represent the gripper's motion in the simulation. Section III explains the cascading optimization method used to produce the final optimal version of the gripper. Section IV highlights the results from the numerical simulations, including some of the simulation's predicted relationships between object reorientation and object size. Section V describes the physical prototypes used to test the results of the parameter optimization, as well as the methods used in the experiments. Section VI concludes the paper.

\section{BACKGROUND}

\section{A. Modeling the GR2 as a Linkage}

A coordinate-free predictor-corrector technique was used to trace the coupler curve of the GR2 Linkage. The coordinatefree approach was chosen because of its advantages over more traditional coordinate dependent methods. Perhaps the greatest advantage is that the equation representing the motion of the linkage can be obtained in a straightforward way, using distance geometry. Simple inspection of the linkage is all that is required, and the equation of motion follows directly. A second advantage is that the equation representing the motion of the linkage can be decomposed into multiple branches, each representing the linkage's motion for a certain configuration [12]. One of these configurations represents the actual motion that is possible for a physical linkage-one that cannot selfintersect-narrowing our focus to a single formula without worry for sign changes.

Given two intersecting circles of known radii, and the distance separating their centers, we can easily calculate the coordinates of their intersection points. This technique, referred to as bilateration, is similar to triangulation but depends only on distances rather than angles. Suppose there are two vectors $\mathbf{p}_{A, B}$ and $\mathbf{p}_{A, C}$ connecting points $A$ to $B$, and points $A$ to $C$. Then there exists a bilateration matrix $\mathbf{Z}_{A, B, C}$ relating these two vectors which can be written in terms of squared distances between the three points $A, B$, and $C$.

$$
\begin{gathered}
\mathbf{p}_{A, C}=\mathbf{Z}_{A, B, C} \mathbf{p}_{A, B} \\
\mathbf{Z}_{A, B, C}=\frac{1}{2 s_{A, B}}\left[\begin{array}{cc}
s_{A, C}+s_{A, B}-s_{B, C} & -4 A_{A, B, C} \\
4 A_{A, B, C} & s_{A, C}+s_{A, B}-s_{B, C}
\end{array}\right]
\end{gathered}
$$

$$
A_{A, B, C}= \pm \frac{1}{4} \sqrt{\left(s_{A, C}+s_{A, B}+s_{B, C}\right)^{2}-2\left(s_{A, C}^{2}+s_{A, B}^{2}+s_{B, C}^{2}\right)}
$$

As an example, $s_{A, C}$ is the squared distance between $A$ and $C$.

$$
l_{A, C}=\sqrt{s_{A, C}}
$$

Notice that $A_{A, B, C}$ can be either positive or negative. This term is known as the oriented area, and its sign is dependent

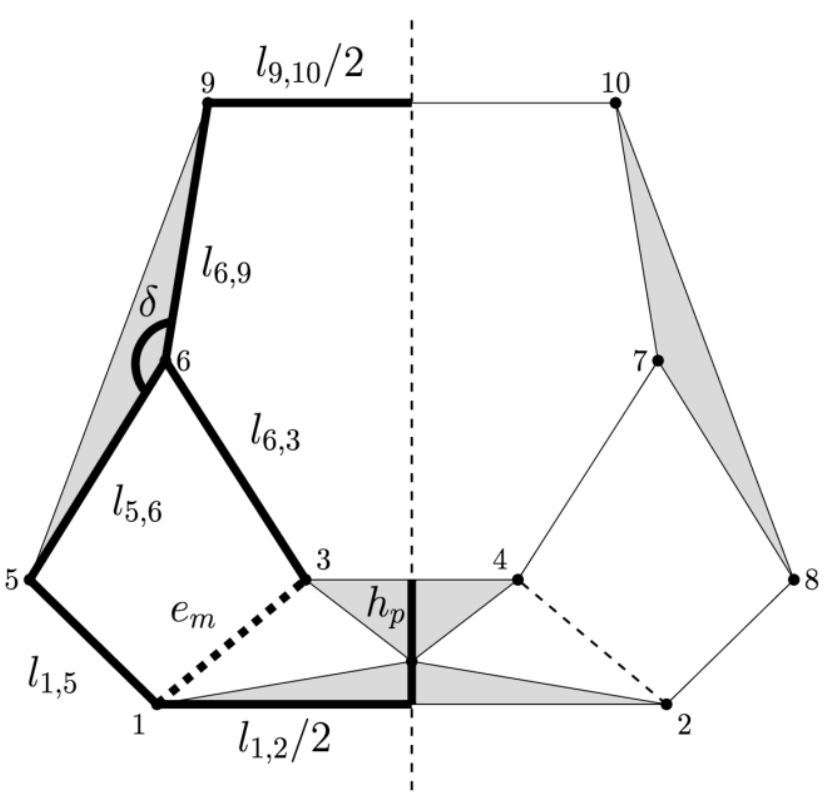

Figure 2. The GR2 gripper can be modeled as an Innocenti Linkage. Heavy black lines indicate link lengths and other parameters which were varied as part of the brute force parameter search. Here, the gripper is shown in its initial pose, with symmetry across the central dashed line.

on the orientation of $\mathbf{p}_{A, C}$ relative to $\mathbf{p}_{A, B}$. If $C$ is to your left as you look straight down vector $\mathbf{p}_{A, B}$, then the oriented area term $A_{A, B, C}$ is positive; otherwise it is negative. A detailed derivation of these formulae and their properties can be found in [13].

Now, we can use bilateration to construct an equation representing the kinematics of the GR2's underlying linkagethe Innocenti linkage-in terms of two variables. Specifically, we are representing the motion of the GR2 in terms of two virtual links, which change length depending on the orientation of the linkage. The general form of the Innocenti linkage - modified to contain a central pivot - is illustrated in Figure 2.

$$
\mathbf{p}_{9,10}=\mathbf{p}_{1,4}+\mathbf{p}_{4,7}+\mathbf{p}_{7,10}-\mathbf{p}_{6,9}-\mathbf{p}_{1,6}
$$

Now, use bilateration matrices to rewrite each vector in terms of the virtual link $\mathbf{p}_{1,6}$. One example is shown below.

$$
\begin{gathered}
\mathbf{p}_{1,4}=\mathbf{Z}_{1,3,4} \mathbf{p}_{1,3} \\
\mathbf{p}_{1,3}=\mathbf{Z}_{1,6,3} \mathbf{p}_{1,6} \\
\Rightarrow \mathbf{p}_{1,4}=\mathbf{Z}_{1,3,4} \mathbf{Z}_{1,6,3} \mathbf{p}_{1,6}
\end{gathered}
$$

Combining all such equations, the result is the following.

$$
\begin{gathered}
\mathbf{p}_{9,10}=\left(-\mathbf{I}+\mathbf{Z}_{1,3,4} \mathbf{Z}_{1,6,3}-\mathbf{Z}_{4,2,7} \mathbf{Z}_{4,1,2} \mathbf{Z}_{1,3,4} \mathbf{Z}_{1,6,3}\right. \\
\left.+\mathbf{Z}_{6,5,9} \mathbf{Z}_{6,1,5}-\mathbf{Z}_{7,8,10} \mathbf{Z}_{7,2,8}\left(\mathbf{I}-\mathbf{Z}_{4,2,7}\right) \mathbf{Z}_{4,1,2} \mathbf{Z}_{1,3,4} \mathbf{Z}_{1,6,3}\right) \mathbf{p}_{1,6}
\end{gathered}
$$

Using the fact that 


$$
\left\|\mathbf{p}_{2}\right\|^{2}=\operatorname{det}(\mathbf{Z})\left\|\mathbf{p}_{1}\right\|^{2}
$$

the vector equation can be rewritten in terms of squared distances. The following equation will be referred to as the closure condition, and is at the core of this simulation.

$$
\begin{gathered}
\Omega=f\left(s_{1,6}, s_{2,7}\right)=\operatorname{det}\left(-\mathbf{I}+\mathbf{Z}_{1,3,4} \mathbf{Z}_{1,6,3}\right. \\
-\mathbf{Z}_{4,2,7} \mathbf{Z}_{4,1,2} \mathbf{Z}_{1,3,4} \mathbf{Z}_{1,6,3}+\mathbf{Z}_{6,5,9} \mathbf{Z}_{6,1,5} \\
\left.-\mathbf{Z}_{7,8,10} \mathbf{Z}_{7,2,8}\left(\mathbf{I}-\mathbf{Z}_{4,2,7}\right) \mathbf{Z}_{4,1,2} \mathbf{Z}_{1,3,4} \mathbf{Z}_{1,6,3}\right)-\frac{s_{9,10}}{s_{1,6}}
\end{gathered}
$$

After obtaining the equation of motion, a Newton-Raphson method was used to numerically approximate its roots, yielding the coupler curve of the linkage

Given two intersecting circles of known radii, and the distance separating their centers, we can easily calculate the coordinates of their intersection points. This technique, referred to as bilateration, is similar to triangulation but depends only on distances rather than angles. Suppose there are two vectors $\mathbf{p}_{A, B}$ and $\mathbf{p}_{A, C}$ connecting points $A$ to $B$, and points $A$ to $C$. Then there exists a bilateration matrix $\mathbf{Z}_{A, B, C}$ relating these two vectors which can be written in terms of squared distances between the three points $A, B$, and $C$.

$$
\begin{gathered}
\mathbf{p}_{A, C}=\mathbf{Z}_{A, B, C} \mathbf{p}_{A, B} \\
\mathbf{Z}_{A, B, C}=\frac{1}{2 s_{A, B}}\left[\begin{array}{cc}
s_{A, C}+s_{A, B}-s_{B, C} & -4 A_{A, B, C} \\
4 A_{A, B, C} & s_{A, C}+s_{A, B}-s_{B, C}
\end{array}\right]
\end{gathered}
$$

$$
A_{A, B, C}= \pm \frac{1}{4} \sqrt{\left(s_{A, C}+s_{A, B}+s_{B, C}\right)^{2}-2\left(s_{A, C}^{2}+s_{A, B}^{2}+s_{B, C}^{2}\right)}
$$
C.

As an example, $s_{A, C}$ is the squared distance between $A$ and

$$
l_{A, C}=\sqrt{s_{A, C}}
$$

Notice that $A_{A, B, C}$ can be either positive or negative. This term is known as the oriented area, and its sign is dependent on the orientation of $\mathbf{p}_{A, C}$ relative to $\mathbf{p}_{A, B}$. If $C$ is to your left as you look straight down vector $\mathbf{p}_{A, B}$, then the oriented area term $A_{A, B, C}$ is positive; otherwise it is negative. A detailed derivation of these formulae and their properties can be found in [13].

Now, we can use bilateration to construct an equation representing the kinematics of the GR2's underlying linkagethe Innocenti linkage-in terms of two variables. Specifically, we are representing the motion of the GR2 in terms of two virtual links, which change length depending on the orientation of the linkage. The general form of the Innocenti linkage - modified to contain a central pivot - is illustrated in Figure 2.

$$
\mathbf{p}_{9,10}=\mathbf{p}_{1,4}+\mathbf{p}_{4,7}+\mathbf{p}_{7,10}-\mathbf{p}_{6,9}-\mathbf{p}_{1,6}
$$

Now, use bilateration matrices to rewrite each vector in terms of the virtual link $\mathbf{p}_{1,6}$. One example is shown below.

$$
\begin{aligned}
\mathbf{p}_{1,4} & =\mathbf{Z}_{1,3,4} \mathbf{p}_{1,3} \\
\mathbf{p}_{1,3} & =\mathbf{Z}_{1,6,3} \mathbf{p}_{1,6} \\
\Rightarrow \mathbf{p}_{1,4} & =\mathbf{Z}_{1,3,4} \mathbf{Z}_{1,6,3} \mathbf{p}_{1,6}
\end{aligned}
$$

Combining all such equations, the result is the following.

$$
\begin{gathered}
\mathbf{p}_{9,10}=\left(-\mathbf{I}+\mathbf{Z}_{1,3,4} \mathbf{Z}_{1,6,3}-\mathbf{Z}_{4,2,7} \mathbf{Z}_{4,1,2} \mathbf{Z}_{1,3,4} \mathbf{Z}_{1,6,3}\right. \\
\left.+\mathbf{Z}_{6,5,9} \mathbf{Z}_{6,1,5}-\mathbf{Z}_{7,8,10} \mathbf{Z}_{7,2,8}\left(\mathbf{I}-\mathbf{Z}_{4,2,7}\right) \mathbf{Z}_{4,1,2} \mathbf{Z}_{1,3,4} \mathbf{Z}_{1,6,3}\right) \mathbf{p}_{1,6}
\end{gathered}
$$

Using the fact that

$$
\left\|\mathbf{p}_{2}\right\|^{2}=\operatorname{det}(\mathbf{Z})\left\|\mathbf{p}_{1}\right\|^{2}
$$

the vector equation can be rewritten in terms of squared distances. The following equation will be referred to as the closure condition, and is at the core of this simulation.

$$
\begin{gathered}
\Omega=f\left(s_{1,6}, s_{2,7}\right)=\operatorname{det}\left(-\mathbf{I}+\mathbf{Z}_{1,3,4} \mathbf{Z}_{1,6,3}\right. \\
-\mathbf{Z}_{4,2,7} \mathbf{Z}_{4,1,2} \mathbf{Z}_{1,3,4} \mathbf{Z}_{1,6,3}+\mathbf{Z}_{6,5,9} \mathbf{Z}_{6,1,5} \\
\left.-\mathbf{Z}_{7,8,10} \mathbf{Z}_{7,2,8}\left(\mathbf{I}-\mathbf{Z}_{4,2,7}\right) \mathbf{Z}_{4,1,2} \mathbf{Z}_{1,3,4} \mathbf{Z}_{1,6,3}\right)-\frac{s_{9,10}}{s_{1,6}}
\end{gathered}
$$

After obtaining the equation of motion, a Newton-Raphson method was used to numerically approximate its roots, yielding the coupler curve of the linkage.

\section{B. Coupler Curve Tracing Algorithm}

A predictor-corrector technique was used to numerically approximate the roots of our closure condition-roots that map directly to the coupler curve of the linkage in Cartesian space. Since the closure condition is a bivariate function, we can use the multivariate Newton-Raphson method to find its roots, as described in Gomes et al. [14]. This process begins by predicting the next value of $s_{1,6}$ by numerically approximating the curve's tangent at the latest known point. This is done using central difference partial derivative approximations, by evaluating the closure condition in a grid around the last known values of $s_{1,6}$ and $s_{2,7}, \pm 0.01$. The initial prediction for the next value of $s_{1,6}$ is just the latest value, plus a small step along the tangent line. The prediction is corrected by repeating this process, using the predicted value as a new starting point, iterating until the difference between two successive predicted values is within the desired accuracy. Next, the lengths of the two elastic elements $s_{1,3}$ and $s_{2,4}$ are calculated, by rotating the central pivot by a small angle $\zeta$ proportional to the difference between $s_{1,6}$ and $s_{2,7}$, such that the elastic links either grow or shrink according to their adjacent virtual link, shown in Figure 


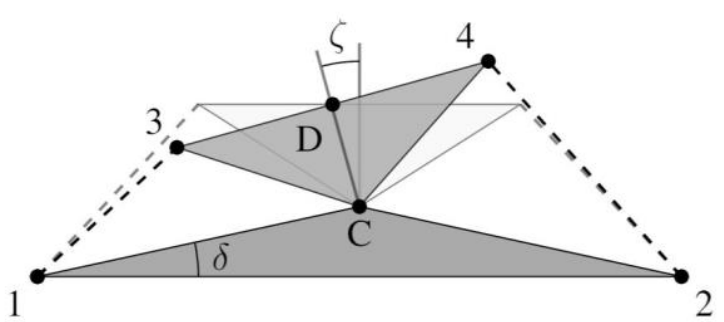

Figure 3. The general form of the elastic base is shown here. The triangular ternary link formed by points 3,4 , and $\mathrm{C}$ is the central pivot, which rotates about $\mathrm{C}$, coupling the motion of both fingers. GR2 grippers with elastic pivots were included in the search after finding that they improved reorientation in the first simulation

3. Finally, the Newton-Raphson process is repeated for $s_{2,7}$, completing one cycle of root approximation.

Prior to tracing the coupler curve of the GR2, we must compute a starting configuration given a set of link length parameters. This is necessary because the numerical curve tracing algorithm needs a starting point (initial values for $l_{1,6}$ and $l_{2,7}$ ) to inform its prediction of the next set of roots of the curve. This is accomplished by starting the grasper from a symmetric initial orientation, meaning that both fingers are mirror images of each other, and that $l_{9,10}$ and $l_{3,4}$ are horizontal and parallel to $l_{1,2}$. We exploit the grasper's underlying geometry under the assumption of symmetry to derive a function relating the object size $s_{9,10}$, to the virtual link $s_{1,6}$, which we can be found using MATLAB's vpasolve for instance. Because of the symmetry assumption, $s_{1,6}$ is initially equal to $S_{2,7}$, and the initial pose is fully defined.

During each tracing cycle, the algorithm checks if various constraints are met-constraints which serve to both realistically limit the motion of the grasper and to vastly reduce the program's runtime. Some constraints, such as joint limits and hard stops, are imposed to reflect the physical conditions under which a real GR2 must operate, and are based on those of the original GR2 model. Specifically, the joint angle created by $l_{1,5}$ and $l_{6,5}$ cannot be smaller than $0.65 \mathrm{rad}$ due to a physical hard stop near the joint. Also, the angle between links $l_{1,2}$ and $l_{1,5}$ cannot exceed $3.30 \mathrm{rad}$ without link $l_{1,5}$ colliding with the top surface of the hand.

A friction cone constraint was also coded into the simulation, assuming a friction coefficient of 1 for rubber on solids between a finger and object. This friction coefficient is conservative, as the material combination of rubber on solids has an estimated friction coefficient in the range of 1-4 according to [15]. This creates a friction cone of $0.785 \mathrm{rad}$ about the axis of each finger's surface normal at the point of contact. Because the GR2 is modeled as a closed linkage, the contact forces must be collinear, otherwise a net torque would be applied to the object, breaking the assumption of quasistatic manipulation. Thus, if the fingers configure in such a way that the line connecting the contact forces moves outside of the friction cones, this constraint is violated and the tracing ends for that grasper.

Constraints were also used to increase the speed of convergence of the search algorithm, such as one that sets the sum of the total inner finger length to a constant value. This constraint maintains the flavor of the original GR2, keeping it from simply growing the size of all parameters. But most importantly, this constraint lowers the dimensionality of the parameter space from 10 to 9 . For example, setting the length of $l_{3,4}$ automatically determines the length of $l_{6,3}$ with this constraint, leaving only $l_{1,5}$, and $l_{6,5}$ to be determined.

Finally, a constraint was created to avoid cases where the Newton-Raphson method did not converge. It is well known that the Newton-Raphson method may fail to converge if either the initial guess is far from the root, or if the derivative of the curve is very small or vanishes [14]. This constraint limited the number of iterations possible for finding each root, and if that number was exceeded, the tracing was halted and the grasper's trajectory was not computed any further.

A predictor-corrector technique was used to numerically approximate the roots of our closure condition-roots that map directly to the coupler curve of the linkage in Cartesian space. Since the closure condition is a bivariate function, we can use the multivariate Newton-Raphson method to find its roots, as described in Gomes et al. [14]. This process begins by predicting the next value of $s_{1,6}$ by numerically approximating the curve's tangent at the latest known point. This is done using central difference partial derivative approximations, by evaluating the closure condition in a grid around the last known values of $s_{1,6}$ and $s_{2,7}, \pm 0.01$. The initial prediction for the next value of $s_{1,6}$ is just the latest value, plus a small step along the tangent line. The prediction is corrected by repeating this process, using the predicted value as a new starting point, iterating until the difference between two successive predicted values is within the desired accuracy. Next, the lengths of the two elastic elements $s_{1,3}$ and $s_{2,4}$ are calculated, by rotating the central pivot by a small angle $\zeta$ proportional to the difference between $s_{1,6}$ and $s_{2,7}$, such that the elastic links either grow or shrink according to their adjacent virtual link, shown in Figure 3. Finally, the Newton-Raphson process is repeated for $s_{2,7}$, completing one cycle of root approximation.

Prior to tracing the coupler curve of the GR2, we must compute a starting configuration given a set of link length parameters. This is necessary because the numerical curve tracing algorithm needs a starting point (initial values for $l_{1,6}$ and $l_{2,7}$ ) to inform its prediction of the next set of roots of the curve. This is accomplished by starting the grasper from a symmetric initial orientation, meaning that both fingers are mirror images of each other, and that $l_{9,10}$ and $l_{3,4}$ are horizontal and parallel to $l_{1,2}$. We exploit the grasper's underlying geometry under the assumption of symmetry to derive a function relating the object size $s_{9,10}$, to the virtual link $s_{1,6}$, which we can be found using MATLAB's vpasolve for instance. Because of the symmetry assumption, $s_{1,6}$ is initially equal to $s_{2,7}$, and the initial pose is fully defined.

During each tracing cycle, the algorithm checks if various constraints are met-constraints which serve to both realistically limit the motion of the grasper and to vastly reduce the program's runtime. Some constraints, such as joint limits and hard stops, are imposed to reflect the physical conditions under which a real GR2 must operate, and are based on those of the original GR2 model. Specifically, the joint angle created by $l_{1,5}$ and $l_{6,5}$ cannot be smaller than 0.65rad due to a physical hard stop near the joint. Also, the angle between links $l_{1,2}$ and $l_{1,5}$ cannot exceed $3.30 \mathrm{rad}$ without link $l_{1,5}$ colliding with the top surface of the hand.

A friction cone constraint was also coded into the simulation, assuming a friction coefficient of 1 for rubber on 
solids between a finger and object. This friction coefficient is conservative, as the material combination of rubber on solids has an estimated friction coefficient in the range of 1-4 according to [15]. This creates a friction cone of $0.785 \mathrm{rad}$ about the axis of each finger's surface normal at the point of contact. Because the GR2 is modeled as a closed linkage, the contact forces must be collinear, otherwise a net torque would be applied to the object, breaking the assumption of quasistatic manipulation. Thus, if the fingers configure in such a way that the line connecting the contact forces moves outside of the friction cones, this constraint is violated and the tracing ends for that grasper.

Constraints were also used to increase the speed of convergence of the search algorithm, such as one that sets the sum of the total inner finger length to a constant value. This constraint maintains the flavor of the original GR2, keeping it from simply growing the size of all parameters. But most importantly, this constraint lowers the dimensionality of the parameter space from 10 to 9 . For example, setting the length of $l_{3,4}$ automatically determines the length of $l_{6,3}$ with this constraint, leaving only $l_{1,5}$, and $l_{6,5}$ to be determined.

Finally, a constraint was created to avoid cases where the Newton-Raphson method did not converge. It is well known that the Newton-Raphson method may fail to converge if either the initial guess is far from the root, or if the derivative of the curve is very small or vanishes [14]. This constraint limited the number of iterations possible for finding each root, and if that number was exceeded, the tracing was halted and the grasper's trajectory was not computed any further.

\section{OPTIMIZATION METHOD}

A brute force search was used to find the grasper parameters that maximized the object reorientation. Specifically, we searched for the grasper that maximized the pure rotation undergone by a grasped object. In total, 9 parameters were varied including $l_{1,5}, l_{5,6}, l_{6,3}, l_{6,9}, l_{9,10}, l_{1,2}, e_{m}$ (elasticity),$\delta$ and $h_{p}$. First, this search was performed with the original GR2 topology to find the link dimensions resulting in the largest possible reorientation. After running this search, it became clear that the width of the palm $l_{3,4}$ was inversely proportional to the maximum achievable reorientation of the hand. This realization prompted another run of the simulation - this time with a modified topology where joints three and four (see Figure 2) come together coaxially, in effect the original GR2 topology with a zero width palm. We reran the simulation with the new coalesced topology, this time searching the space around the best grasper from the first search. From here on out, we will refer to the graspers as 'original', 'intermediate', and 'optimal coalesced'. The physical versions of these grippers are shown in Figure 6.

\section{NUMERICAL RESULTS}

The predicted achievable reorientation of the best grippers from each brute force search are shown in Figure 4. The intermediate GR2 is the best gripper out of over 20,000 unique graspers. The optimal coalesced GR2 was the best out of over 3,000 graspers - fewer than the previous simulation because the coalescence of the palm joints removes a parameter from the search space. In general, the simulations predict better performance from elastic central pivots than from locked pivots. The maximum achievable reorientation for each grasper peaks at a particular object size, then decreases for larger objects. As successive grippers are able to achieve higher ranges of reorientation, the object size at which the maximum reorientation occurs becomes smaller. For example, the simulation predicts that the optimal coalesced gripper reaches maximum reorientation for objects around $25 \mathrm{~mm}$ in size, while the original gripper achieves its maximum for objects close to $48 \mathrm{~mm}$ across. Overall, the maximum achievable reorientation predicted by the simulation is slightly above $1.5 \mathrm{rad}$, for objects $25 \mathrm{~mm}$ across, with the elastic pivot on the optimal coalesced gripper.

\section{PROTOTYPE AND EXPERIMENT}

Three physical GR2 models were built with dimensions equal to the best gripper found from each subsequent brute force parameter space search, shown in Figures 5 and 6. Model parts were printed on a Stratasys Fortus $250 \mathrm{mc}$, and joints consisted of precision ground steel pins press fitted into the model. High friction finger pads with ridges were molded using Smooth-On Vytaflex ${ }^{\circledR} 30$, shown in Figure 5. The achievable reorientation of each model was tested using a "sweep" motion primitive from the OpenHand [16] software repository, that moves a grasped object from one end of the
Original GR2

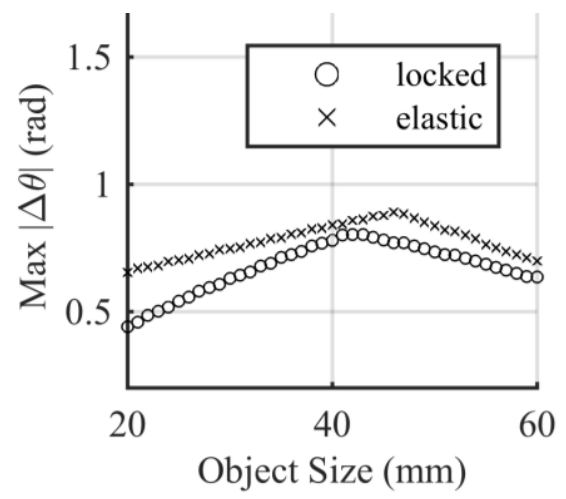

Intermediate GR2

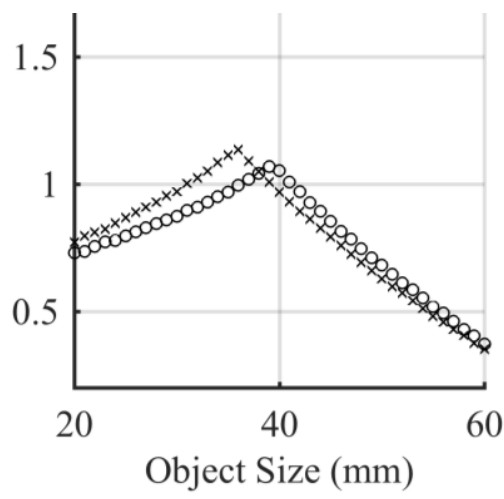

Optimal Coalesced GR2

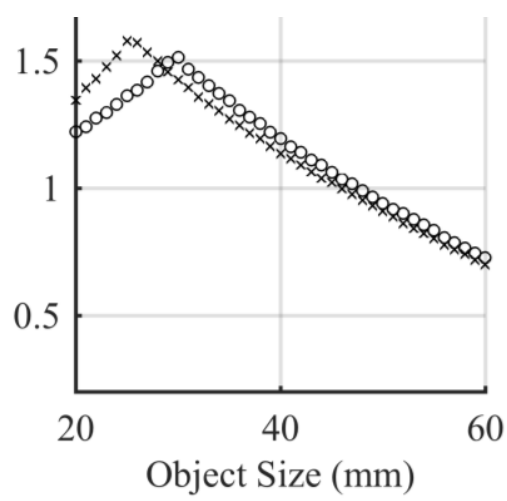

Figure 4. Predicted reorientation range for all three variations of the GR2 from the simulation. Left: the original GR2; Center: the intermediate GR2; Right: the optimal coalesced GR2 


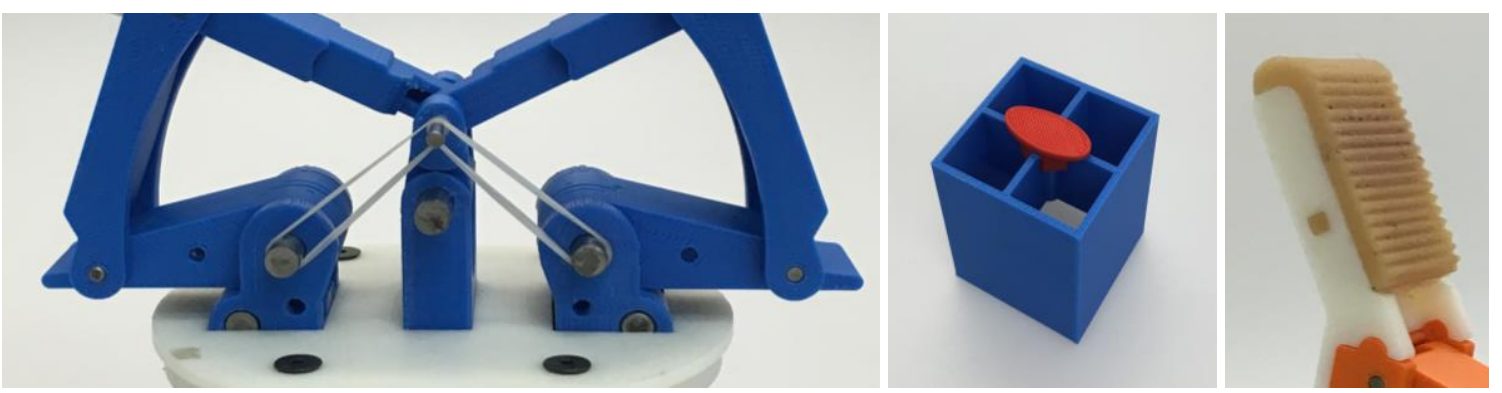

Figure 5. Left: the GR2 gripper's central pivot is shown with elastic bands. In some cases, an elastic pivot produced more reorientation than a locked pivot; Middle: an object is shown with an elliptical red marker attached to its center. These markers were used to track the object's position and orientation; Right: the gripper's high friction finger pads are shown in detail.
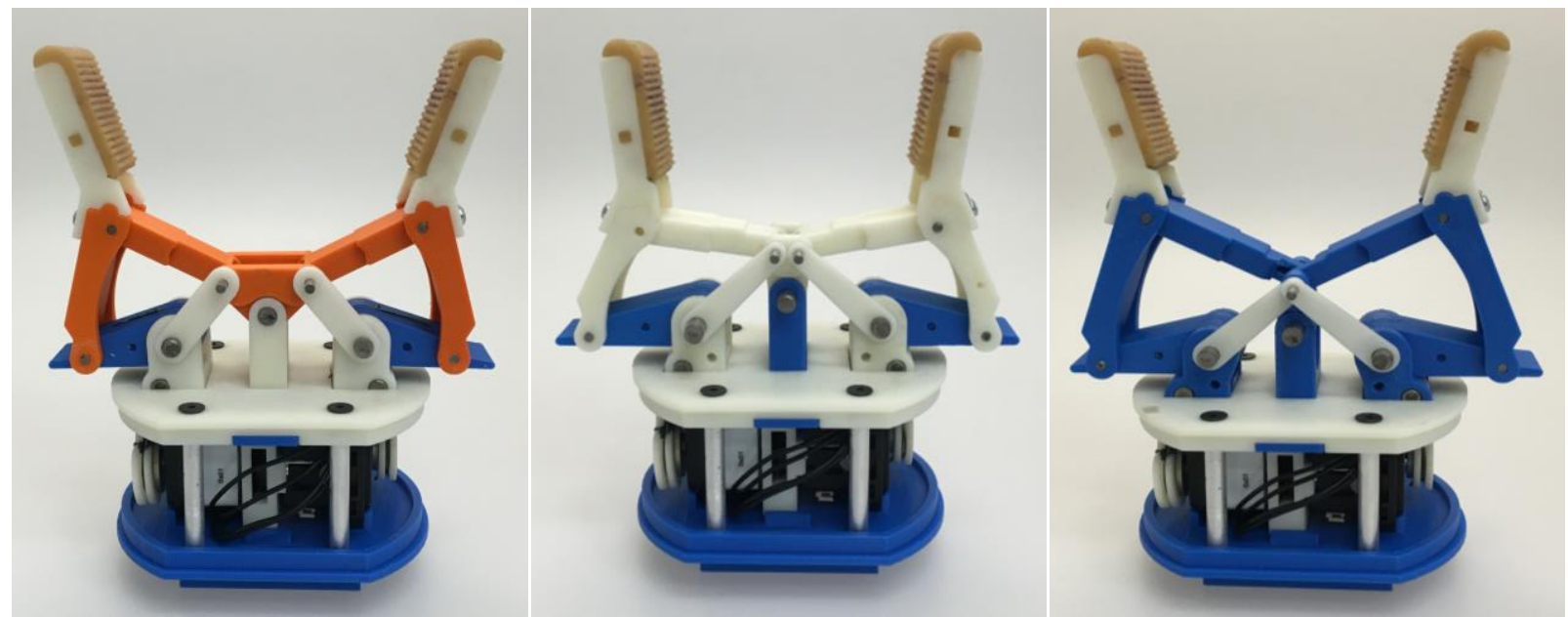

Figure 6. Left: the original GR2; Center: the intermediate GR2; Right: the optimal coalesced GR2. The reorientation capabilities of the original GR2 improve as the width of its palm decreases, resulting in its best form, the intermediate GR2. Taking this trend even further, the brute force parameter search was repeated with a new topology—with the palm joints coalesced into a single coaxial joint, shown on the right.

grasper's workspace to the other. Specifically, this means there is a pushing finger operating in position control mode, and an opposing holding finger operating in torque control mode. The position controlled finger pushed the object as far to the opposite side of the workspace as possible, while the torque controlled finger maintains a holding grasp on the object. Then, the mode of each finger swaps, and the object is pushed as far as possible to the opposite side of the workspace. This motion is repeated multiple times. This was performed both with elastic and rigidly supported central pivots for all graspers. Five square objects and five circular objects were manipulated, ranging in size from $20 \mathrm{~mm}$ to $63 \mathrm{~mm}$ across. Square objects were manipulated first with corners between the fingertips, then with sides flat against the fingertips. A red ellipse was attached to the center of each object and tracked using a Logitech C920 webcam and MATLAB's built in motion tracking functions. In this way, object position and orientation data was recorded in real time during manipulation testing. The object was placed at the same location within the fingertips prior to each trial, to ensure consistent results - even though most objects could be successfully manipulated over a large range of initial positions within the fingertips, due to the reorientation being largely a result of fingertip shearing motion. Once the object was manually loaded into the correct position within the fingertips, a secure grasp command was executed, the object tracking software was launched, and the trial proceeded. In total, data was collected for 90 unique handobject-pivot style combinations. A trial was considered successful if the gripper did not eject the object, and if it was able to sweep the object back and forth through its workspace 5 times without loss of contact. Ejection occurred rarely-only when the object orientation caused the line of force transmission between contacts to fall outside of the friction cones. Assuming a strong initial grasp, objects tended to remain grasped during manipulation. The results of these experiments are shown in Figure 7. First, the difference between the best overall grasper - the coalesced optimal GR2 with the locked pivot - and the original grasper with the locked pivot is shown, as improvement in reorientation. Also shown are the achievable reorientations for each hand-objectpivot combination from the experiments. Figure 8 compares the achievable reorientation of the locked original GR2 to the locked optimal coalesced GR2, for both the simulation and experimental results, over a range of object sizes. Specifically, it shows the experimental results from the square corners based manipulation.

\section{CONCLUSION}

The exploration of the parameter space was vast enough to find an improved gripper for each hand topology, at least within a region around the initial gripper dimensions. The initial simulation predicted that grippers with smaller palms should outperform those with wide palms, resulting in the intermediate gripper. Following this intuition, we modified the topology of the gripper, bringing the palm together into a single coalesced joint - a ternary joint with two joints sharing 

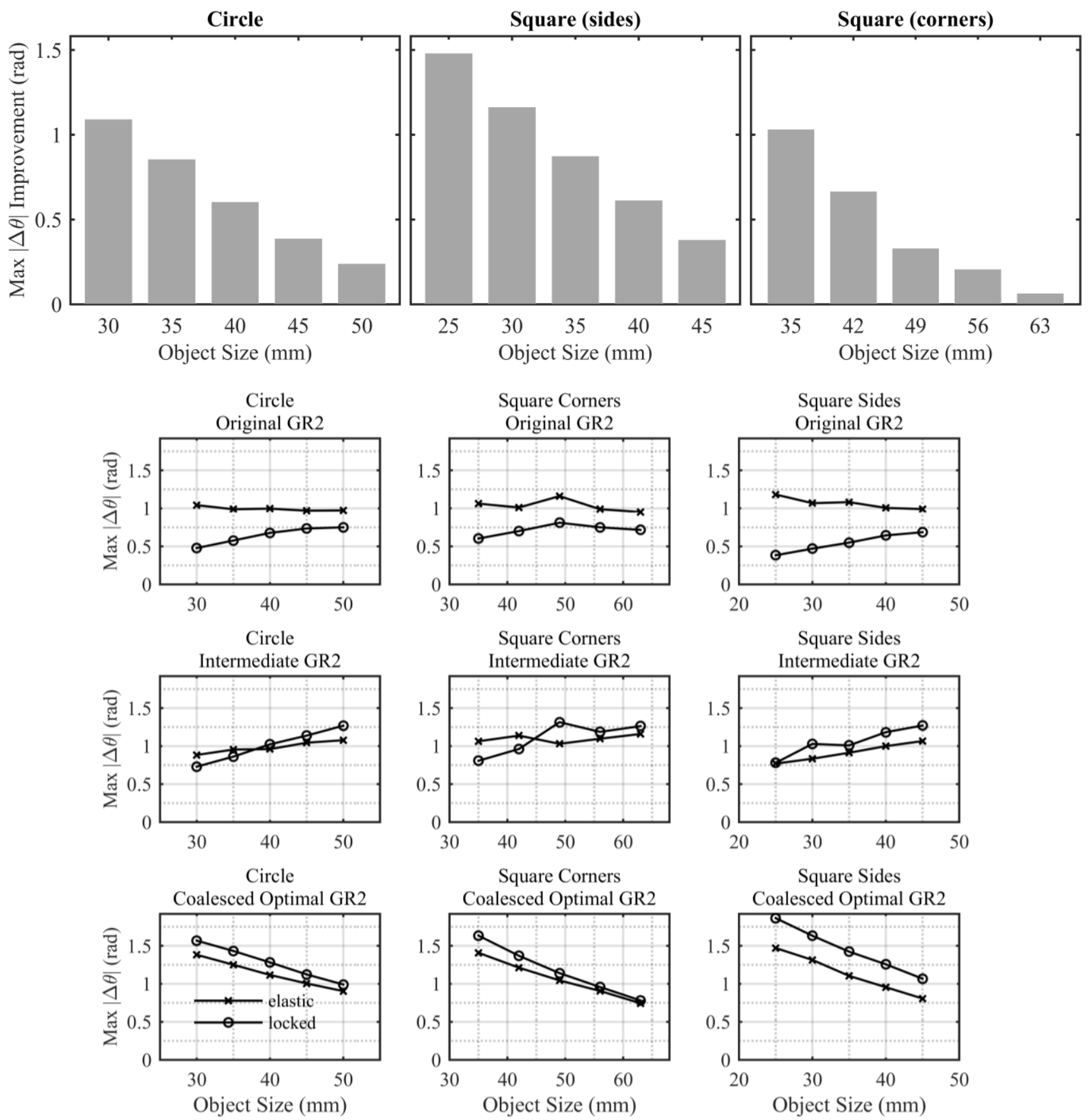

Figure 7. Top three plots: The improvement in the achievable reorientation between the coalesced GR2 gripper and the original GR2 gripper, both with locked central pivot. All other plots show the absolute achievable reorientation of each hand object combination from the experiments.

an axis, and reran the simulation. Once again, the results were even better than before. The simulation predicted reorientation of over 1.5rad for the optimal coalesced hand topology. Each gripper was physically constructed and tested experimentally by sweeping objects from one end of the hand workspace to the other, pushing the actuators to their maximum torque. In all, data was collected for 90 unique test cases, consisting of 15 different hand object combinations, 2 styles of central pivot support, and 3 different graspers. The experimental results agree with the simulation. Any differences in achievable reorientation can be attributed to complicated contact conditions not accounted for in the simulation, such as rolling and slip, and to oversimplified predictions for the behavior of the elastic links.

\section{ACKNOWLEDGMENT}

The authors would like to thank Raymond R. Ma, Minas Liarokapis, and Neil Bajaj for their technical assistance and the fruitful discussions held during the preparation of this paper. 


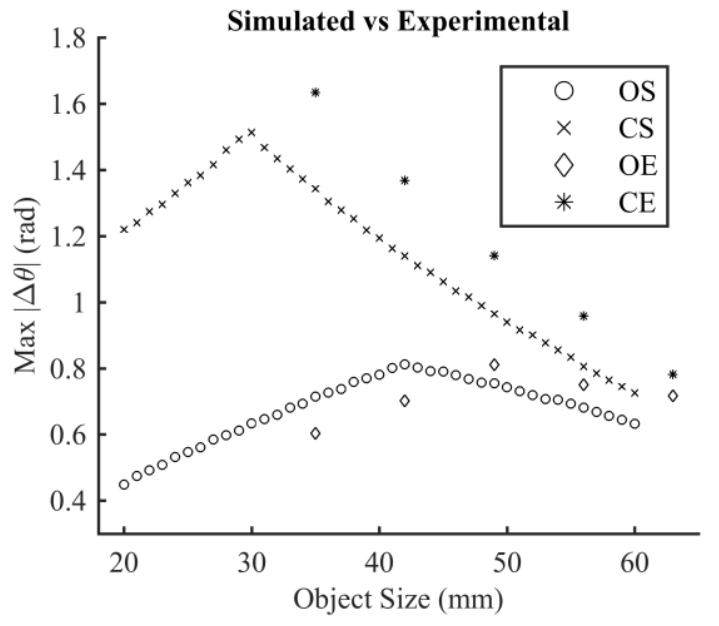

Figure 8. The simulated and experimental results for both the locked original and locked coalesced grippers. This experimental data is from manipulation of a square object, by the corners. OS: original GR2 simulated. CS: coalesced optimal GR2 simulated. OE: original GR2 experimental. CE: coalesced optimal GR2 experimental.

\section{REFERENCES}

[1] G. O. Young, "Synthetic structure of industrial plastics (Book style with paper title and editor)," in Plastics, 2nd ed. vol. 3, J. Peters, Ed. New York: McGraw-Hill, 1964, pp. 15-64.

[2] W.-K. Chen, Linear Networks and Systems (Book style). Belmont, CA: Wadsworth, 1993, pp. 123-135.

[3] H. Poor, An Introduction to Signal Detection and Estimation. New York: Springer-Verlag, 1985, ch. 4.

[4] B. Smith, "An approach to graphs of linear forms (Unpublished work style)," unpublished.

[5] E. H. Miller, "A note on reflector arrays (Periodical style-Accepted for publication)," IEEE Trans. Antennas Propagat., to be published.

[6] J. Wang, "Fundamentals of erbium-doped fiber amplifiers arrays (Periodical style-Submitted for publication)," IEEE J. Quantum Electron., submitted for publication.

[7] C. J. Kaufman, Rocky Mountain Research Lab., Boulder, CO, private communication, May 1995.

[8] Y. Yorozu, M. Hirano, K. Oka, and Y. Tagawa, "Electron spectroscopy studies on magneto-optical media and plastic substrate interfaces(Translation Journals style)," IEEE Transl. J. Magn.Jpn., vol. 2, Aug. 1987, pp. 740-741 [Dig. , $^{\text {th }}$ Annu. Conf. Magnetics Japan, 1982, p. 301].

[9] M. Young, The Techincal Writers Handbook. Mill Valley, CA: University Science, 1989.

[10] J. U. Duncombe, "Infrared navigation-Part I: An assessment of feasibility (Periodical style)," IEEE Trans. Electron Devices, vol. ED11, pp. 34-39, Jan. 1959.

[11] S. Chen, B. Mulgrew, and P. M. Grant, "A clustering technique for digital communications channel equalization using radial basis function networks," IEEE Trans. Neural Networks, vol. 4, pp. 570578, July 1993.

[12] R. W. Lucky, "Automatic equalization for digital communication," Bell Syst. Tech. J., vol. 44, no. 4, pp. 547-588, Apr. 1965.

[13] S. P. Bingulac, "On the compatibility of adaptive controllers (Published Conference Proceedings style)," in Proc. 4th Annu. Allerton Conf. Circuits and Systems Theory, New York, 1994, pp. 8 16.

[14] G. R. Faulhaber, "Design of service systems with priority reservation," in Conf. Rec. 1995 IEEE Int. Conf. Communications, pp. 3-8.

[15] W. D. Doyle, "Magnetization reversal in films with biaxial anisotropy," in 1987 Proc. INTERMAG Conf., pp. 2.2-1-2.2-6.
[16] G. W. Juette and L. E. Zeffanella, "Radio noise currents n short sections on bundle conductors (Presented Conference Paper style)," presented at the IEEE Summer power Meeting, Dallas, TX, June 2227, 1990, Paper 90 SM 690-0 PWRS.

[17] J. G. Kreifeldt, "An analysis of surface-detected EMG as an amplitudemodulated noise," presented at the 1989 Int. Conf. Medicine and Biological Engineering, Chicago, IL.

[18] J. Williams, "Narrow-band analyzer (Thesis or Dissertation style)," Ph.D. dissertation, Dept. Elect. Eng., Harvard Univ., Cambridge, MA, 1993.

[19] N. Kawasaki, "Parametric study of thermal and chemical nonequilibrium nozzle flow," M.S. thesis, Dept. Electron. Eng., Osaka Univ., Osaka, Japan, 1993.

[20] J. P. Wilkinson, "Nonlinear resonant circuit devices (Patent style)," U.S. Patent 3624 12, July 16, 1990. 